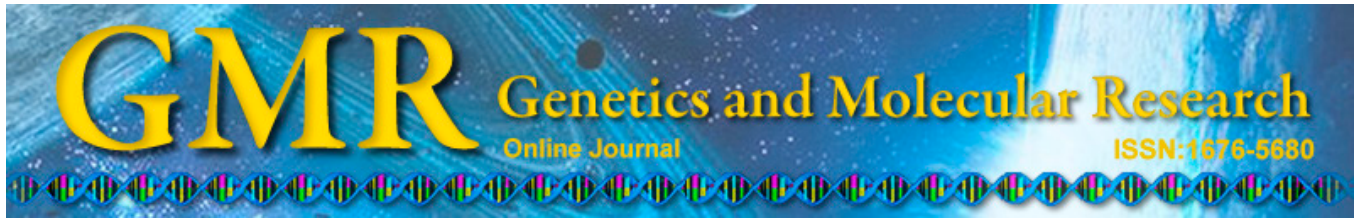

\title{
Effects of propofol and etomidate pretreatment on glucocorticoid receptor expression following induction of sepsis in rats
}

\author{
C. Wang ${ }^{1}$, N. Liu ${ }^{1}$, R.-M. Li ${ }^{1}$, Y. Zhang ${ }^{1}$, L. Shen ${ }^{2}$ and J.-Y. Xiong ${ }^{1}$ \\ ${ }^{1}$ Department of Anesthesiology, The Second Affiliated Hospital, \\ Dalian Medical University, Dalian, Liaoning, China \\ ${ }^{2}$ Laboratory of Molecular Biology, The Second Affiliated Hospital, \\ Dalian Medical University, Dalian, Liaoning, China
}

Corresponding author: J.-Y. Xiong

E-mail: jyxiong0638@163.com

Genet. Mol. Res. 14 (2): 4740-4748 (2015)

Received July 11, 2014

Accepted November 18, 2014

Published May 11, 2015

DOI http://dx.doi.org/10.4238/2015.May.11.6

\begin{abstract}
The aim of this study was to determine the effect of etomidate and propofol pretreatment on the expression of glucocorticoid receptor and the prognosis of sepsis. The sepsis rat was used as a model. During glucocorticoid treatment, etomidate and propofol were applied alone or together at different time points. Survival curves, glucocorticoid receptor expression in the rat adrenal cortex, and inflammation levels were determined. The outcome of sepsis in rats was evaluated based on the combined utilization of propofol and etomidate. The results indicated that the combined utilization of propofol and etomidate pretreatment could significantly improve the effects of glucocorticoids on rat sepsis. Etomidate was shown to enhance the expression of the glucocorticoid receptor, while propofol was shown to inhibit the inflammatory response. Etomidate was best used immediately after modeling, whereas propofol was most suitable for use during the peak inflammatory reaction. These results demonstrated that anesthetics had
\end{abstract}


the ability to enhance the effect of glucocorticoids in the treatment of sepsis. Etomidate was indicated for use in the early stage of inflammation to enhance expression of the glucocorticoid receptor, while propofol application was indicated at the peak of the inflammatory reaction owing to its strong anti-inflammation effect.

Key words: Propofol; Etomidate; Sepsis; Glucocorticoid receptor

\section{INTRODUCTION}

Sepsis and the subsequent sepsis shock, multiple organ dysfunction syndrome (MODS), and other symptoms, represent one of the most common causes of death of infected critically ill patients and infants (Pejo et al., 2012). At present, research on the pathogenesis of sepsis has concluded that the adrenal cortex plays an important role in inhibiting the excessive damage from inflammation during sepsis. The prognosis of patients with sepsis and adrenocortical insufficiency is often poor (Desai et al., 2013). Glucocorticoid (GC) treatment of severe sepsis is currently the main measure for clinical rescue. However, individual patients exhibit different responses to hormonal treatment because of differences in glucocorticoid receptor (GR) expression level and extent (Sneyd, 2012). To address this issue, we investigated adrenal GR expression and evaluated the efficacy of hormone therapy in sepsis by using a rat model.

\section{MATERIAL AND METHODS}

\section{Sepsis rat modeling}

Female Sprague-Dawley rats were used in this study at 8-12 weeks old, weighing 280 $\pm 15 \mathrm{~g}$. All animals were housed and maintained at a specific-pathogen-free grade animal center.

Rats were fasted for $12 \mathrm{~h}$ prior to surgery and were allowed water ad libitum. Cecal ligation and puncture (CLP) rats were anaesthetized by administration of $10 \%$ chloral hydrate. Surgery was conducted under aseptic conditions. An incision was made through the skin and abdominal wall lateral to the midline and the cecum was exposed and immobilized. A 5-0 sterile surgical suture ligation was placed on the cecum at a position approximately one-third distance from the blind side. A needle was passed through the anterior and posterior walls of the cecum in one single motion twice and the cecum was gently squeezed to ensure patency of the puncture wounds and to extrude a small amount of fecal content. The cecum was returned to the peritoneal cavity and the abdominal incision was closed in layers. CLP rats received $2 \mathrm{~mL} 0.9 \%$ sterile $\mathrm{NaCl}$ intraperitoneally after surgery to help replace water loss from the operation and promote the speed of spreading of intestinal contents. Following surgery, the rats were returned to their cages and were closely monitored. Venous cannula was given to the rats and fixed at the neck skin.

Indication of rat sepsis included: agitation, chills, abdominal inflation, increase of eye secretions, and piloerection. Abdominal hemorrhagic effusions, bowel flatulence, and empyema in the bowel or kidney were visible upon postmortem examination.

\section{Animal grouping}

Rats were grouped according to the different processing method and observational 
target as follows: Group I: blank group (control), rats with no treatment; group II: treatment control group, rats with sham surgery; group III: sepsis model group, rats treated to induce sepsis; Group IV: GC treatment group, rats received immediate postoperative low-dose (6 mg/ $\mathrm{kg}$ ) hydrocortisone.

After confirmation of the therapeutic effect in groups I-IV, rats in groups V and VI received etomidate or propofol on the basis of group IV. Group V: V1, etomidate injury group, rats received etomidate $6 \mathrm{~h}$ after the surgery at $2 \mathrm{mg} \cdot \mathrm{kg}^{-1} \cdot \mathrm{h}^{-1}$ through the jugular vein for $2 \mathrm{~h}$; $\mathrm{V} 2$, etomidate pretreatment group, rats received immediate postoperative etomidate at $1 \mathrm{mg} /$ $\mathrm{kg}$; group VI: VI1, propofol injury group, rats received propofol $6 \mathrm{~h}$ after the surgery at 24 $\mathrm{mg} \cdot \mathrm{kg}^{-1} \cdot \mathrm{h}^{-1}$ through the jugular vein for $2 \mathrm{~h}$; VI2, propofol pretreatment group, rats received immediate postoperative etomidate at $10 \mathrm{mg} / \mathrm{kg}$.

\section{Sample collection}

\section{Blood specimen collection}

Blood samples $(500 \mu \mathrm{L})$ were collected pre-operatively and at $6,12,24$, and $48 \mathrm{~h}$ after the surgery. The blood specimens were precooled at $4^{\circ} \mathrm{C}$ for $2 \mathrm{~h}$, then centrifuged for 5 $\min$ at $1400 \mathrm{~g}$ and $4^{\circ} \mathrm{C}$. The supernatant (serum) was centrifuged for $10 \mathrm{~min}$ at $14,000 \mathrm{~g}$ and $4^{\circ} \mathrm{C}$ and stored at $-80^{\circ} \mathrm{C}$. Hemolytic blood specimens were rejected. Inflammatory factors and renal function index in the blood specimens were tested by an enzyme-linked immunoassay (ELISA) kit (USCN, Wuhan, China).

\section{Tissue specimen collection}

Rats were sacrificed under aseptic conditions. Immediately following, the adrenal glands were collected and surface attachments were removed. The medulla were separated and discarded in the aseptic culture dish, while the left cortex was collected for RNA extraction.

Total RNA was isolated from the adrenal cortex by TRIzol (TaKaRa Bio, Otsu, Shiga, Japan), and cDNA was synthesized using reverse transcriptase (Takara) after the RNA quality was verified by a spectrometer. Quantitative real-time polymerase chain reaction (qPCR) amplification was performed and detected on an ABI Stepone system (Applied Biosystems, Inc., Foster City, CA, USA). The cycling conditions consisted of an initial single cycle of $5 \mathrm{~min}$ at $94^{\circ} \mathrm{C}$, followed by 35 cycles of $45 \mathrm{~s}$ at $94^{\circ} \mathrm{C}, 45 \mathrm{~s}$ at $55^{\circ} \mathrm{C}$, and $60 \mathrm{~s}$ at $72^{\circ} \mathrm{C}$. The primers used are listed in Table 1 .

\begin{tabular}{ll}
\multicolumn{2}{c}{ Table 1. PCR primers used in this study. } \\
\hline Gene name & Sequence $5^{\prime}-3^{\prime}$ \\
\hline GR & F: ACT GCA GGA GTC TCA CAA GAC AC \\
& R: TGT CTG GAA GCA GTA GGT AAG GA \\
\multirow{2}{*}{$J n k-1$} & F: ATC TCC CCC AAC TTC AGC TT \\
& R: TGA TGG GGC TTT GAA GGT AG \\
& F: AAC GAC CTT CTA CGA CGA TG \\
& R: GCA GCG TAT TCT GGC TAT GC \\
\hline
\end{tabular}




\section{Observation targets}

\section{Rat survival status}

The survival status and time of death of the rats in each group were recorded for generation of the survival curve.

\section{GC-related receptor expression analysis}

qPCR was used to measure the expression level of the GR. Flow cytometry was used to analyze the GR expression differences between the freshly isolated adrenal cortex cells. Furthermore, $M k p-1$ and Jnk expression levels were also detected by qPCR.

\section{Rat serum inflammatory factor test}

Interleukin (IL)-1 and tumor necrosis factor (TNF)- $\alpha$ expression levels were determined in serum samples collected prior to surgery and at $6,12,24$, and $48 \mathrm{~h}$ after surgery.

\section{Statistical analysis}

Alterations between different subgroups were analyzed by the chi-square and Student $t$-tests, as appropriate. A survival curve was used for survival analysis. A P value $<0.05$ was used to indicate statistical significance. All statistical analyses were performed using the SPSS17.0 software (SPSS, Chicago, IL, USA).

\section{RESULTS}

\section{Rat survival analysis}

After the surgery, rats in the blank and control groups were found to have normal survival. All of the rats in the model group died within 10 days after modeling, and the time of death was primarily concentrated between the 2 nd and 5 th days. This result suggested that severe inflammation in the early stage of sepsis was the main cause of death. After GC treatment, the death rate fell to $70 \%$, and the time of death was delayed, which implied that the inhibitory impact of GC treatment was related to the early inflammatory response. However, a 70\% mortality rate also revealed that a single GC treatment did not confer substantial improvements. The rat death rate declined further after etomidate and propofol incorporation. In the F2 group, with immediate usage of propofol after surgery, and the E1 group, with administration of etomidate $6 \mathrm{~h}$ after surgery, rat mortality dropped to $30 \%$. Compared with groups F1 and E2, groups F2 and E1 had better curative effects, which suggested that they comprised better drug-delivery methods. The survival curve is shown in Figure 1. 
A

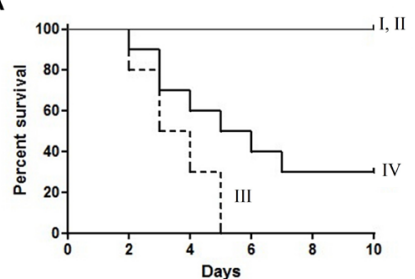

B

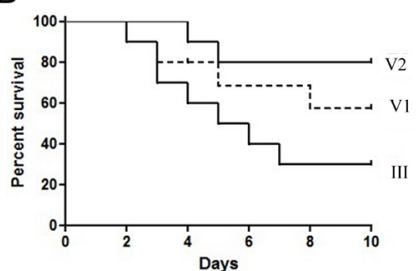

C

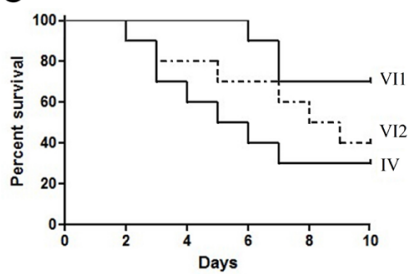

Figure 1. Rat survival analysis. A. Rat survival status after GC treatment: I: blank group; II: control group. No deaths were observed in these two groups within the 10-day observation period. Group III was the modeling group; deaths occurred beginning at the 2 nd day after surgery and no rats survived past the 5 th day. Group IV was the GC treatment group; one rat died every day from the 2 nd to the 7 th day after surgery and three animals survived to the 10th day. B. Rat survival status after etomidate treatment: Rats in group V exhibited enhanced survival as compared with group IV. Specifically, the death rate from sepsis declined from the 5th day after surgery following immediate postoperative etomidate usage, which implied that etomidate was more effective than was GC in controlling sepsis. C. Rat survival status after propofol treatment: Rats in group VI survived better than those in group IV. Specifically, the death rate from sepsis declined from the 2nd to 3rd day after surgery following propofol administration, which implied that the effect of propofol was stronger than that of $\mathrm{GC}$ when used $6 \mathrm{~h}$ after surgery.

\section{Expression of rat adrenal cortex receptors and pathway proteins}

RNA was extracted from adrenal cortices for qPCR analysis $12 \mathrm{~h}$ after sepsis modeling. GR expression in the sepsis model rat significantly decreased when compared with normal controls, while the inflammation signaling protein gene $M k p-1$ was upregulated after sepsis. Furthermore, Jnk, which has been shown to mediate inflammation and facilitate cell apoptosis, was also activated in the sepsis rat. After GC treatment, GR expression decreased more obviously. However, expression of the inflammation indicator $M \mathrm{kp}-1$ and apoptosis indicator $J n k$ was also inhibited. This result suggested the dual characteristic of GC therapy, as it not only appeared to inhibit inflammation and cell apoptosis, but also further decreased the organisms' reactivity to subsequent GC treatment.

After further combined usage of anesthetic, it was observed that immediate postoperative propofol usage could decrease $M k p-1$ and $J n k$ expression significantly $(\mathrm{P}<0.05)$. Furthermore, immediate use of GR after surgery could remarkably enhance GR expression levels. This result suggested that propofol had a marked anti-inflammatory effect in sepsis therapy, while etomidate improved the GC function significantly.

The result of drug administration at different time points implied that, for propofol, MKP-1 and JNK inhibition was more affected when the drug was administrated at $6 \mathrm{~h}$ after surgery. However, for etomidate, GR expression did not show a significant difference $(\mathrm{P}=$ 0.521 ) between the two drug-delivery methods. These phenomena also suggested that propofol might better protect the viscera when used at the peak of inflammation, while earlier use of etomidate might be preferred for GC treatment (Figure 2).

\section{Analysis of rat inflammatory status}

There were no observed differences in IL-1 and TNF- $\alpha$ between the blank and control groups, suggesting that a simple laparotomy does not lead to significant acute inflammatory stress response. However, IL-1 and TNF- $\alpha$ presented a significant peak at 6-12 $\mathrm{h}$ after surgery in the modeling group $(\mathrm{P}<0.01)$. On the other hand, though the inflammatory peak in group 
D was higher than that of the control, it was notably decreased compared with the modeling group (Figure 3A and B).

For all the rats treated with $\mathrm{GC}$ and narcotic drugs, postoperative inflammation markedly decreased after using propofol, while the effects of etomidate were not as significant ( $\mathrm{P}$ $<0.05$ ) (Figure 3C and D).

A

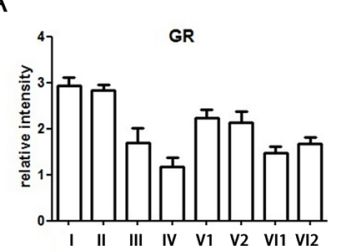

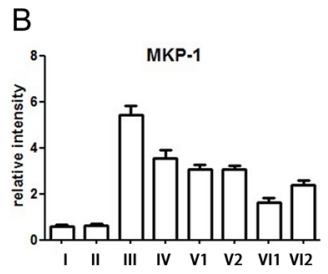

C

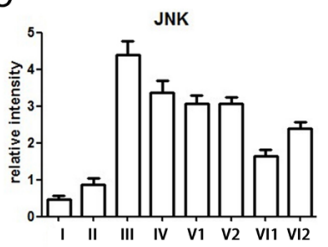

Figure 2. Expression of rat adrenal cortex receptors and pathway proteins (means $\pm \mathrm{SE}$ ). A. GR expression in the different groups. GR expression was similar in the blank and control groups (I, II). GR expression in the modeling group decreased, and it decreased further after GC treatment. This decrease correlated with the GR feedback down regulation that occurred under HPA axis activation during sepsis. GR expression recovered slightly after propofol was administered and this accorded with its the anti-inflammatory effects. However, different dosing time had similar effects on GR expression (VI1, VI2). The GR expression level increased significantly after etomidate involvement $(\mathrm{P}=0.035)$; furthermore, immediate dosing after surgery had a stronger effect than dosing after $6 \mathrm{~h}$. GR: B. MKP-1 expression in the different groups. MKP-1 was activated in the modeling group, while its activation status was inhibited after GC usage (D). Combined usage of etomidate or propofol further decreased MK-1 expression (E, F). Of the two, propofol had the better therapeutic effect, and the inhibition effect was enhanced when the drug was administered at $6 \mathrm{~h}$ after surgery $(\mathrm{F} 1, \mathrm{~F} 2)$. C. JNK expression in the different groups. JNK was activated in the modeling group, while its activation status was inhibited after GC usage (D). Combined usage of etomidate or propofol further decreased MK-1 expression (E, F). Of the two drugs, propofol had better therapeutic effect, and the inhibition effect was enhanced when administered at $6 \mathrm{~h}$ after surgery (F1, F2).

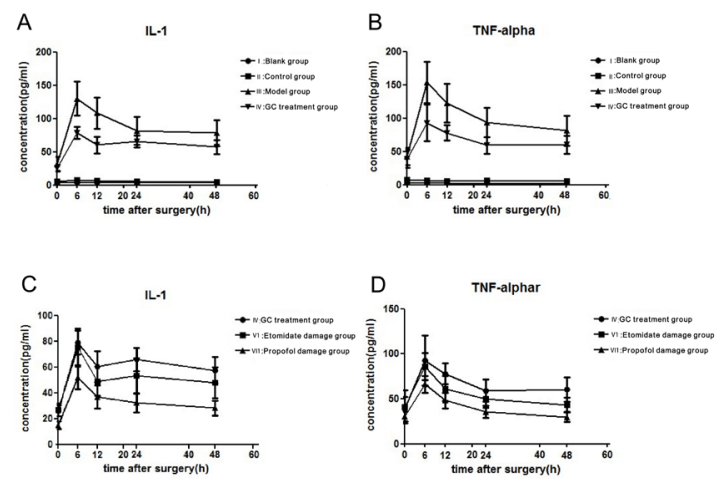

Figure 3. Analysis of rat inflammatory status. A. Blood IL-1 expression in rat sepsis. IL-1 expression in serum was stable in both treatment and control groups. It appeared to be rapidly improved and was maintained at a high level in the modeling group. Specifically, it reached the peak of inflammation at 6 to $12 \mathrm{~h}$ after surgery. The inflammation amplitude was relieved by administration of GC. B. Blood TNF- $\alpha$ expression in rat sepsis. TNF- $\alpha$ expression in serum was stable in the treatment and control groups. It appeared to be rapidly improved and was maintained at a high level in the modeling group. Specifically, it reached the peak of inflammation at 6 to $12 \mathrm{~h}$ after surgery. The inflammation amplitude was relieved by administration of GC. C. IL-1 expression based on propofol and etomidate usage at 6 $\mathrm{h}$ after surgery. The IL-1 proliferation curve could be lowered by GC treatment combined with either propofol or etomidate. The propofol anti-IL-1 effect was greater than that of etomidate. D. TNF- $\alpha$ expression based on propofol and etomidate usage at $6 \mathrm{~h}$ after surgery. The TNF- $\alpha$ proliferation curve could be lowered by GC treatment combined with either propofol or etomidate. The propofol anti-TNF- $\alpha$ effect was greater than that of etomidate. 


\section{DISCUSSION}

In the clinic, sepsis is seen most often in cases of severe trauma, burns, infection, and major surgical complications (Forman, 2011). Clinical epidemiology data show that the mortality rate from sepsis is between 30 and 50\% (van den Heuvel et al., 2013). The main cause of death is the injury of acute internal organs and the respiratory system by severe inflammation. Previous animal studies and clinical observation have confirmed that the pituitary gland-hypothalamus-adrenal axis (HPA axis) is the most important mechanism for the body's resistance to sepsis. However, in the condition of sepsis, inflammatory mediators can inhibit the function of the adrenal cortex resulting in loss of the cortex reserve capacity. Meanwhile, these inflammatory cytokines can change the expression and function of GR by inducing overexpression of $M K P-1$ and $J N K$, resulting in a reduction in the number of GRs and concomitant declined affinity for GC (Chen et al., 2010). Patients with sepsis, therefore, often exhibit relative adrenocortical insufficiency, which is an important reason for the less significant therapeutic effect of single-use GC.

In this study, we successfully simulated the process of sepsis infection after major surgery using the appendix perforation operation model. We observed manifestations of septic shock such as chills, abdominal distension, and canthus secretion after surgery. Based on this, we successfully prolonged the postoperative survival time of rats through the use of GC. The results were consistent with clinical observation (Yazid et al., 2010). On the other hand, under the sepsis condition, inflammatory reaction-related signals were overexpressed in rats, and GR expression also increased. The change was correlated with the HPA activation and septic shock status. However, though the inflammatory reaction signals were reduced after GC treatment, the expression of GRs also decreased. Though a large amount of exogenous GC can counteract the impact of GR decline totally, single GC therapy might reduce the body's response to the exogenous GC, and might therefore expect to yield better curative effects with improved GR expression following the introduction of GC (Perretti and D'Acquisto, 2009; Wancket et al., 2012).

In recent years, studies have shown that anesthetic drugs can inhibit the immune response and control the exudation of inflammatory mediators (Horlacher et al., 2011). Propofol, a type of widely used anesthetic, had been noted for its anti-inflammatory effects. It was found that propofol could effectively inhibit inflammation caused by endotoxin. Serum levels of inflammatory factors such as IL-6 and TNF- $\alpha$ were significantly decreased upon propofol treatment (Ko et al., 2009; Hammer et al., 2010). Further cell experiments also suggested that propofol could stabilize the cell membrane and reduce inflammatory factors secreted by macrophages and T cells released in the inflammation. In addition, propofol has the most minimal impact on the endocrine system among anesthetics (Tamura et al., 2002). However, propofol can substantially inhibit the respiratory and circulatory systems. Therefore, the rescue of sepsis through propofol use in the clinic still had great theoretical risk. Many other drugs can also be used for anti-inflammation effects; thus, the clinical application of propofol still lacks powerful physiological and pharmacological support (Esposito et al., 2012). Considering the question of cardiovascular safety, etomidate has received more attention for its reduced impact on cardiovascular function and stable hemodynamics (He et al., 2012). A recent study reported that intravenous etomidate also had good anti-inflammatory effects. Although some researchers pointed out that etomidate might partly inhibit adrenal cortex secretion, such inhibition could be compensated for by exogenous cortical hormone. The goal for treatment with any 
drug, of course, would be for the actual therapeutic effect to provide more benefit than harm to the patient.

In the present study, we adopted the method of propofol and etomidate pretreatment to observe the effects on $\mathrm{GC}$ treatment for the control of sepsis in rats. In comparison with the single GC treatment, we found the survival time of rats to be extended by post-operative propofol and etomidate administration. Thus, combined application of GC and anesthetics can achieve better anti-sepsis effects than GC application alone. Previous research had pointed out that this effect is caused by the anti-inflammatory peroxide damage and cell membrane stabilization effect of anesthetics (Ayroldi et al., 2012). In our study, we found a significant reduction in inflammatory factors concentrations in serum under propofol and etomidate treatment, while the GR expression was increased (Ayroldi et al., 2012). This result demonstrates that these two types of anesthetics can improve the outcome of sepsis in animals by inhibiting inflammation and increasing GR levels.

Though the combination of propofol and etomidate can improve the effect of sepsis treatment, there are big differences between the methods and times of delivery of the anesthetic in clinical application (Goodwin et al., 2013). We tried different dosing strategies aimed to assess the best delivery time and method for propofol and etomidate administration. Ultimate survival, GR expression, and the concentration of serum inflammatory factors were used as indices for assessing the effects of the different strategies.

For propofol, though the inflammatory factors were elevated compared with the immediate postoperative group, rats in the propofol injury group had better ultimate survival and renal function. This result showed that dosing with propofol at $6 \mathrm{~h}$ after surgery is preferred over immediate postoperative treatment. Therefore, propofol showed a significant anti-inflammatory effect, both in the previous and present studies. However, inflammation in the early stage of sepsis still had positive relevance for limiting sepsis spread and HPA axis activation (Min et al., 2011; Kleiman et al., 2012). Therefore, the timing propofol dosing to the peak of inflammation might be a better strategy than dosing immediately following surgery. In contrast, the treatment result for etomidate is opposite to that of propofol. Immediate postoperative treatment with etomidate had a better response than did slow pump at $6 \mathrm{~h}$ after surgery. The anti-inflammation ability of etomidate is weaker than that of propofol as shown by the results of inflammatory pathway qPCR and ELISA detection of IL-1 and TNF- $\alpha$. However, etomidate can better stimulate GR expression. Accordingly, early use of etomidate would not be expected to have a marked impact on inflammatory activation of the HPA axis, but could lead to overexpression of GR to enhance the feedback inhibition ability of the HPA axis on inflammatory damage (Smoak and Cidlowski, 2004). Thus, etomidate used immediately after surgery could achieve a better overall effect.

We investigated the similarities and differences between two kinds of drugs in the treatment of sepsis by combined GC therapy and propofol/etomidate pretreatment on sepsis rats. We preliminarily confirmed that anesthetics and corticosteroid combined therapy in the treatment of sepsis had a better treatment effect than did single therapy by evaluating the kidney function, cortical inflammation, cell apoptosis, inflammatory cytokines, and GR levels. We further found that propofol had a strong anti-inflammatory effect and was more suitable for use at the inflammation peak, whereas etomidate could significantly improve the level of GRs, which meant its usage was indicated at the early stage of inflammation. These findings have important relevance for the choice of anesthetic types for combination therapy with GC at different clinical stages. 


\title{
ACKNOWLEDGMENTS
}

\author{
Research supported by the Natural Science Foundation of China (\#81171791).
}

\section{REFERENCES}

Ayroldi E, Cannarile L, Migliorati G, Nocentini G, et al. (2012). Mechanisms of the anti-inflammatory effects of glucocorticoids: genomic and nongenomic interference with MAPK signaling pathways. FASEB J. 26: 4805-20.

Chen Z, Yoshihara E, Son A, Matsuo Y, et al. (2010). Differential roles of Annexin A1 (ANXA1/lipocortin-1/lipomodulin) and thioredoxin binding protein-2 (TBP-2/VDUP1/TXNIP) in glucocorticoid signaling of HTLV-I-transformed T cells. Immunol. Lett. 131: 11-8.

Desai R, Miller KW and Raines DE (2013). The pyrrole etomidate analog carboetomidate potently inhibits human 5-HT3A receptor function: comparisons with etomidate and potential implications for emetogenesis. Anesth. Analg. 116: 573-9.

Esposito E, Bruscoli S, Mazzon E, Paterniti I, et al. (2012). Glucocorticoid-induced leucine zipper (GILZ) over-expression in T lymphocytes inhibits inflammation and tissue damage in spinal cord injury. Neurotherapeutics. 9: 210-25.

Forman SA (2011). Clinical and molecular pharmacology of etomidate. Anesthesiology 114: 695-707.

Goodwin JE, Feng Y, Velazquez H, Sessa WC, et al. (2013). Endothelial glucocorticoid receptor is required for protection against sepsis. Proc. Natl. Acad. Sci. U. S. A. 110: 306-11.

Hammer M, Echtenachter B, Weighardt H, Jozefowski K, et al. (2010). Increased inflammation and lethality of Dusp1-/mice in polymicrobial peritonitis models. Immunology 131: 395-404.

He L, Yang N, Isales CM and Xing-Ming Shi (2012). Glucocorticoid-induced leucine zipper (GILZ) antagonizes TNFalpha inhibition of mesenchymal stem cell osteogenic differentiation. PLoS One 7: e31717.

Horlacher T, Noti C, de Paz JL, P. Bindschädler, et al. (2011). Characterization of annexin A1 glycan binding reveals binding to highly sulfated glycans with preference for highly sulfated heparan sulfate and heparin. Biochemistry 50 : 2650-9.

Kleiman A, Hubner S, Rodriguez Parkitna JM, Neumann A, et al. (2012). Glucocorticoid receptor dimerization is required for survival in septic shock via suppression of interleukin-1 in macrophages. FASEB J. 26: 722-9.

Ko HM, Oh SH, Bang HS, Kang NI, et al. (2009). Glutamine protects mice from lethal endotoxic shock via a rapid induction of MAPK phosphatase-1. J. Immunol. 182: 7957-62.

Min Y, Han D, Fu Z, Honghai Wang, et al. (2011). alpha-MSH inhibits TNF-alpha-induced maturation of human dendritic cells in vitro through the up-regulation of ANXA1. Acta Biochim. Biophys. Sin. 43: 61-8.

Pejo E, Feng Y, Chao W, Cotten JF, et al. (2012). Differential effects of etomidate and its pyrrole analogue carboetomidate on the adrenocortical and cytokine responses to endotoxemia. Crit. Care Med. 40: 187-92.

Perretti M and D'Acquisto F (2009). Annexin A1 and glucocorticoids as effectors of the resolution of inflammation. Nat. Rev. Immunol. 9: 62-70.

Smoak KA and Cidlowski JA (2004). Mechanisms of glucocorticoid receptor signaling during inflammation. Mech. Ageing Dev. 125: 697-706.

Sneyd JR (2012). Novel etomidate derivatives. Curr. Pharm. Des. 18: 6253-6.

Tamura S, Hanada M, Ohnishi M, Katsura K, et al. (2002). Regulation of stress-activated protein kinase signaling pathways by protein phosphatases. Eur. J. Biochem. 269: 1060-6.

van den Heuvel I, Wurmb TE, Bottiger BW and Bernhard M (2013). Pros and cons of etomidate - more discussion than evidence? Curr Opin Anaesthesiol. 26: 404-8.

Wancket LM, Meng X, Rogers LK and Liu Y (2012). Mitogen-activated protein kinase phosphatase (Mkp)-1 protects mice against acetaminophen-induced hepatic injury. Toxicol. Pathol. 40: 1095-105.

Yazid S, Ayoub SS, Solito E, McArthur S, et al. (2010). Anti-allergic drugs and the Annexin-A1 system. Pharmacol. Rep. 62: 511-7. 